\title{
MATRIX OPERATORS AND HYPERINVARIANT SUBSPACES
}

\author{
F. RĂDULESCU AND F.-H. VASILESCU
}

\begin{abstract}
In this paper we study the super-decomposability of some matrix operators as well as some other special properties. These matrix operators are derived from non-analytic functional calculi. As by-products, we obtain statements concerning the existence of (non-trivial) hyperinvariant subspaces.
\end{abstract}

\section{Introduction}

Let $X$ be a complex Banach space and let $\mathscr{L}(X)$ be the algebra of all bounded linear operators acting on $X$. For each $S \in \mathscr{L}(X)$ we denote its spectrum by $\sigma(S)$.

For a fixed integer $n \geqslant 1$, let $X^{n}$ denote the Banach space $X \oplus \ldots \oplus X$ ( $n$ copies). Every operator $T \in \mathscr{L}\left(X^{n}\right)$ can be represented as a matrix $\left(T_{j k}\right)_{j, k=1}^{n}$, where $T_{j k} \in \mathscr{L}(X)$ for each pair of indices $j, k$. We shall study in the sequel a class of operators $T \in \mathscr{L}\left(X^{n}\right)$ with the property that the operators $T_{j k}$ from the matrix representation of $T$ mutually commute. To present this class, we need some preliminaries.

Let $\Omega$ be a compact topological space, let $C(\Omega)$ be the algebra of all complexvalued continuous functions on $\Omega$, and let $A \subset C(\Omega)$ be a (not necessarily closed) subalgebra. We recall that $A$ is said to be normal if, for every open cover $\left\{G_{1}, \ldots, G_{m}\right\}$ of $\Omega$, there are positive functions $f_{1}, \ldots, f_{m}$ in $A$ such that $\operatorname{supp}\left(f_{p}\right) \subset G_{p}(p=1, \ldots, m)$ and

$$
f_{1}(\omega)+\ldots+f_{m}(\omega)=1
$$

for all $\omega \in \Omega$. In particular, $l \in A$ (the positivity of the functions $f_{1}, \ldots, f_{m}$ will play no role in what follows).

1.1 Definition. For an algebra $A \subset C(\Omega)$ we shall consider the following properties :

(i) $A$ is a normal algebra;

(ii) for every pair $f, h \in A$ such that

$$
\operatorname{supp}(h) \subset\{\omega \in \Omega: f(\omega) \neq 0\},
$$

the function $\omega \rightarrow h(\omega) / f(\omega)$, extended with zero outside the set $\operatorname{supp}(h)$, is an element of $A$;

(iii) $A$ has a Banach algebra structure which makes the inclusion $A \subset C(\Omega)$ continuous.

We shall indicate at the beginning of each section which of these hypotheses on the algebra $A$ are going to be used.

Received 6 May 1986.

1980 Mathematics Subject Classification 47B40. 
It is clear that $C(\Omega)$ has the properties (i), (ii) and (iii). If $\Omega$ is the closure of a relatively compact open subset of $\mathbb{B}^{m}$, then the algebra $C^{r}(\Omega)$ of all functions $r$-times differentiable in the interior of $\Omega$ whose partial derivatives up to order $r$ have continuous extensions to $\Omega$, also has the properties (i), (ii) and (iii). These are, in fact, the most significant examples that we have in mind.

If $A$ is an arbitrary commutative unital algebra, we denote by $M_{n}(A)$ the algebra of $n \times n$-matrices whose entries are elements of $A$. The algebra $M_{n}(A)$ will sometimes be regarded as an $A$-module. Every unital algebra morphism $\Phi: A \rightarrow \mathscr{L}(X)$ induces a unital algebra morphism $\Phi_{n}: M_{n}(A) \rightarrow \mathscr{L}\left(X^{n}\right)$, defined by the equality $\Phi_{n}(\alpha)=\left(\Phi\left(\alpha_{j k}\right)\right)_{j, k-1}^{n}$, where $\alpha=\left(\alpha_{j k}\right)_{j, k-1}^{n} \in M_{n}(A)$.

1.2 Definition. Let $A \subset C(\Omega)$ be an algebra with the properties (i) and (ii) from Definition 1.1. An operator $T \in \mathscr{L}\left(X^{n}\right)$ will be called $(A, n)$-scalar if there exists a unital algebra morphism $\Phi: A \rightarrow \mathscr{L}(X)$ and an element $\tau \in M_{n}(A)$ such that $T=\Phi_{n}(\tau)$.

This concept extends the concept of $n$-spectral operator, introduced in [9], which in turn extends that of $n$-normal operator [7]. When $A$ is an admissible algebra of continuous functions, then Definition 1.2 also provides an extension of the concept of $A$-scalar operator $[5,12]$ (with respect to this class of admissible algebras).

One of the main purposes of this paper is to prove that every $(A, n)$-scalar operator is super-decomposable [8] (in particular decomposable $[5,12]$ ). Specifically, we shall show that if $T \in \mathscr{L}\left(X^{n}\right)$ is $(A, n)$-scalar and $\left\{U_{1}, U_{2}\right\}$ is an open cover of $\sigma(T)$, then there exists an operator $R \in \mathscr{L}\left(X^{n}\right)$ such that $R T=T R, \sigma\left(T \mid \overline{R\left(X^{n}\right)}\right) \subset U_{1}$ and $\sigma\left(T \mid \overline{\left.\left(1_{n}-R\right)\left(X^{n}\right)\right)} \subset U_{2}\right.$ (where $1_{n}$ is the identity of $X^{n}$; we use the same notation for the identity of $M_{n}(A)$ ). With the terminology of [8], this means precisely that an $(A, n)$-scalar operator is super-decomposable (see Theorem 3.9).

The decomposability of an $(A, n)$-scalar operator $T \in \mathscr{L}\left(X^{n}\right)$ can be used to derive the existence of a proper hyperinvariant subspace (that is, invariant under each operator commuting with $T$ ), when $\sigma(T)$ contains at least two points. This explains one of the main results of [9] (see Corollary 3.7 and Remark 3.8).

By analysing the spectrum of an $(A, n)$-scalar operator $T$ (Theorem 4.6), we shall obtain the existence of hyperinvariant subspaces of $T$, even if $\sigma(T)$ contains only one point, provided that $T$ is not a multiple of the identity; thus we obtain a complete extension of [7, Theorem 5.3] (see Corollary 4.8).

In connection with this subject, we also refer to $[2,6,10,11]$. We can apply our methods to a large enough class of matrix operators, including matrices of generalized scalar operators given by a specral distribution [5].

\section{A spectral capacity}

Let $A \subset C(\Omega)$ be a normal algebra. We also fix a unital algebra morphism $\Phi: A \rightarrow \mathscr{L}(X)$ and denote by $\Phi_{n}$ the corresponding morphism of $M_{n}(A)$ into $\mathscr{L}\left(X^{n}\right)$ induced by $\Phi$.

Since a matrix $\alpha=\left(\alpha_{j k}\right)_{j, k-1}^{n} \subset M_{n}(A)$ can be regarded as a function $\alpha: \Omega \rightarrow M_{n}$ (where $M_{n}=M_{n}(\mathbb{C}) \subset M_{n}(A)$ ), the notation $\alpha(\omega)=\left(\alpha_{j k}(\omega)\right)_{j, k=1}^{n}(\omega \in \Omega)$ and $\operatorname{supp}(\alpha)$ makes sense. Moreover,

$$
\operatorname{supp}\left(\alpha^{\prime} \cdot \alpha^{\prime \prime}\right) \subset \operatorname{supp}\left(\alpha^{\prime}\right) \cap \operatorname{supp}\left(\alpha^{\prime \prime}\right)
$$

for each pair $\alpha^{\prime}, \alpha^{\prime \prime} \in M_{n}(A)$. 
For every $f \in A$ we denote by $\delta(f) \in M_{n}(A)$ the matrix $\delta(f)=\left(\delta_{j k} f\right)_{j, k-1}^{n}$, where $\delta_{j k}$ is the Kronecker symbol. Notice that $\delta$ is, in fact, a unital algebra morphism of $A$ into $M_{n}(A)$ and that $\delta(A)$ is in the centre of $M_{n}(A)$.

The set supp ( $\Phi)$ (that is the support of $\Phi$ ) is defined as the intersection of all closed sets $F \subset \Omega$ such that $\Phi(f)=0$ whenever supp $(f) \subset \Omega \backslash F(f \in A)$. The set $\operatorname{supp}\left(\Phi_{n}\right)$ is defined in a similar way. It is easily seen that $\operatorname{supp}\left(\Phi_{n}\right)=\operatorname{supp}(\Phi)$. (Note that $\operatorname{supp}(\alpha)=\bigcup\left\{\operatorname{supp}\left(\alpha_{j k}\right): 1 \leqslant j, k \leqslant n\right\}$ for each $\alpha=\left(\alpha_{j k}\right)_{j, k-1}^{n} \in M_{n}(A)$.)

2.1 Proposition. For every closed set $F \subset \Omega$ we define the space

$$
X_{\Phi}^{n}(F)=\bigcap\left\{\operatorname{ker}\left(\Phi_{n}(\alpha)\right): \operatorname{supp}(\alpha) \cap F=\varnothing\right\} .
$$

Then the assignment $F \rightarrow X_{\Phi}^{n}(F)$ is a spectral capacity $[3,12]$.

Proof. We follow some lines from the proof of [12, Theorem IV.7.3] (see also [1]).

It is clear that $X_{\Phi}^{n}(F)$ is a closed linear subspace of $X^{n}$. It is easily seen that $X_{\Phi}^{n}(\varnothing)=\{0\}, X_{\Phi}^{n}(\Omega)=X^{n}$ and $X_{\Phi}^{n}\left(F_{1}\right) \subset X_{\Phi}^{n}\left(F_{2}\right)$ whenever $F_{1} \subset F_{2}$.

Let $\left\{G_{1}, \ldots, G_{m}\right\}$ be an open cover of $\Omega$. Since $A$ is normal, we can find functions $f_{1}, \ldots, f_{m}$ in $A$ such that $\operatorname{supp}\left(f_{p}\right) \subset G_{p}(p=1, \ldots, m)$ and $f_{1}+\ldots+f_{m}=1$. Let $\alpha_{p}=\delta\left(f_{p}\right)$; therefore $\operatorname{supp}\left(\alpha_{p}\right) \subset G_{p}$ and $\alpha_{1}+\ldots+\alpha_{m}=1_{n}$. It is then clear that

$$
X^{n}=\Phi_{n}\left(\alpha_{1}\right) X^{n}+\ldots+\Phi_{n}\left(\alpha_{m}\right) X^{n} .
$$

We have only to note that

$$
\Phi_{n}\left(\alpha_{p}\right) X^{n} \subset X_{\Phi}^{n}\left(\operatorname{supp}\left(\alpha_{p}\right)\right) \subset X_{\Phi}^{n}\left(\bar{G}_{p}\right)
$$

for every $p$, and therefore

$$
X^{n}=X_{\Phi}^{n}\left(\bar{G}_{1}\right)+\ldots+X_{\Phi}^{n}\left(\bar{G}_{m}\right)
$$

Now, let $\left\{F_{\gamma}\right\}_{\gamma \in \Gamma}$ be an arbitrary family of closed subsets of $\Omega$. We shall prove that

$$
X_{\Phi}^{n}\left(\bigcup_{\gamma \in \Gamma} F_{\gamma}\right)=\bigcup_{\gamma \in \Gamma} X_{\Phi}^{n}\left(F_{\nu}\right)
$$

Since the mapping $F \rightarrow X_{\Phi}^{n}(F)$ is increasing, it suffices to prove that the right-hand side of (2.3) is contained in the left-hand side. Let $x \in X_{\Phi}^{n}\left(F_{y}\right)$ for all $\gamma \in \Gamma$ and let $F_{0}=\bigcap\left\{F_{\gamma}: \gamma \in \Gamma\right\}$. Let also $\alpha \in M_{n}(A)$ be such that $\operatorname{supp}(\alpha) \cap F_{0}=\varnothing$. Since $\operatorname{supp}(\alpha)$ is compact, we can choose open sets $H_{q}=\Omega \backslash F_{y_{q}}(q=1, \ldots, r)$ such that $\operatorname{supp}(\alpha) \subset H_{1} \cup \ldots \cup H_{r}$. If $H_{0}=\Omega \backslash \operatorname{supp}(\alpha)$, there are functions $h_{0}, h_{1}, \ldots, h_{r}$ in $A$ such that $h_{0}+h_{1}+\ldots+h_{r}=1$ and $\operatorname{supp}\left(h_{q}\right) \subset H_{q}(q=0,1, \ldots, r)$. Let $\beta_{q}=\delta\left(h_{q}\right) \in M_{n}(A)$. Then

$$
\Phi_{n}(\alpha) x=\Phi_{n}\left(\alpha \beta_{0}\right) x+\Phi_{n}\left(\alpha \beta_{1}\right) x+\ldots+\Phi_{n}\left(\alpha \beta_{r}\right) x .
$$

Since $\operatorname{supp}(\alpha) \cap \operatorname{supp}\left(\beta_{0}\right)=\varnothing$ and $\operatorname{supp}\left(\alpha \beta_{q}\right) \cap F_{\gamma_{q}}=\varnothing \quad(1 \leqslant q \leqslant r)$, we have $\Phi_{n}\left(\alpha \beta_{q}\right) x=0$ for all $q=0,1, \ldots, r$. Consequently $\Phi_{n}(\alpha) x=0$, so that $x$ is contained in the left-hand side of (2.3). The proof of Proposition 2.1 is complete.

2.2 Corollary. Let $f \in A$ be such that $\operatorname{supp}(f) \cap \operatorname{supp}(\Phi)=\varnothing$. Then $\Phi(f)=0$.

Proof. Consider first a closed set $F \subset \Omega$ such that if $h \in A$ and $\operatorname{supp}(h) \cap F=\varnothing$, then $\Phi(h)=0$. In this case we must have $X_{\Phi}^{n}(F)=X^{n}$, by (2.1). Indeed, if $\alpha \in M_{n}(A)$ and $\operatorname{supp}(\alpha) \cap F=\varnothing$, then $\Phi_{n}(\alpha)=0$, that is $\operatorname{ker}\left(\Phi_{n}(\alpha)\right)=X^{n}$. 
Now, let $\left\{F_{y}\right\}_{y \in \Gamma}$ be the family of all closed subsets of $\Omega$ sharing the property of $F$. Then $\bigcap\left\{F_{\gamma}: \gamma \in \Gamma\right\}=\operatorname{supp}(\Phi)$. Since $\operatorname{supp}(\delta(f)) \cap \operatorname{supp}(\Phi)=\varnothing$, it follows that

$$
\operatorname{ker}\left(\Phi_{n}(\delta(f))\right) \supset X_{\Phi}^{n}(\operatorname{supp}(\Phi))=\bigcap_{\gamma \in \Gamma} X^{n}\left(F_{y}\right)=X^{n},
$$

by (2.3) and the first part of the proof. Consequently $\Phi(f)=0$.

2.3 COROLlaRY. For every closed $F \subset \Omega$ we have the equality

$$
X_{\Phi}^{n}(F)=X_{\Phi}^{n}(F \cap \operatorname{supp}(\Phi)) .
$$

Proof. As we have noted in the proof of Corollary 2.2,

$$
X_{\Phi}^{n}(\operatorname{supp}(\Phi))=X^{n}
$$

Therefore

by (2.3).

$$
X_{\Phi}^{n}(F)=X^{n} \cap X_{\Phi}^{n}(F)=X_{\Phi}^{n}(F \cap \operatorname{supp}(\Phi)),
$$

2.4 Remark. We have not used so far the fact that the functions of $A$ are continuous.

A supplementary condition on the algebra $A \subset C(\Omega)$ makes the mapping $\Phi_{n}: M_{n}(A) \rightarrow \mathscr{L}\left(X^{n}\right)$ injective on its support.

2.5 Lemma. Assume that the algebra A also has the property (ii) of Definition 1.1. If $\Phi_{n}(\alpha)=0$ for some $\alpha \in M_{n}(A)$, then $\alpha(\omega)=0$ for every $\omega \in \operatorname{supp}(\Phi)$.

Proof. Note first that if $\Phi(f)=0$ for some $f \in A$, then $f(\omega)=0$ for every $\omega \in \operatorname{supp}(\Phi)$.

Indeed, if $h \in A$ is such that $\operatorname{supp}(h) \subset G=\{\omega \in \Omega: f(\omega) \neq 0\}$, then the extension $h_{1}$ of the function $\omega \mapsto h(\omega) / f(\omega)$ belongs to $A$ and we have $\Phi(h)=\Phi\left(h_{1}\right) \Phi(f)=0$. Therefore $\operatorname{supp}(\Phi) \cap G=\varnothing$.

Now, if $\Phi_{n}(\alpha)=0$ and $\alpha=\left(\alpha_{j k}\right)_{j, k-1}^{n}$, then $\Phi\left(\alpha_{j k}\right)=0$ for each pair $(j, k)$. By the previous remark, it follows that $\alpha(\omega)=0$ for all $\omega \in \operatorname{supp}(\Phi)$.

\section{Decomposability}

In this section $A$ will denote a subalgebra of $C(\Omega)$ with the properties (i) and (ii) from Definition 1.1. Let $\Phi: A \rightarrow \mathscr{L}(X)$ be a fixed initial algebra morphism. We also fix an element $\tau=\left(\tau_{j k}\right)_{j, k=1}^{n} \in M_{n}(A)$. Let $T=\Phi_{n}(\tau) \in \mathscr{L}\left(X^{n}\right)$, that is, $T$ is $(A, n)$-scalar. From the defining relation (2.1), it follows easily that $T X_{\Phi}^{n}(F) \subset X_{\Phi}^{n}(F)$ for all closed subsets $F \subset \Omega$.

3.1 Lemma. For every closed $F \subset \Omega$ we have the inclusion

$$
\sigma\left(T \mid X_{\Phi}^{n}(F)\right) \subset \bigcup_{\omega \in F} \sigma(\tau(\omega)),
$$

and the set on the right-hand side is closed. 
Proof. We use the well-known equality

$$
\sigma(\tau(\omega))=\left\{z \in \mathbb{C}: \operatorname{det}\left(z 1_{n}-\tau(\omega)\right)=0\right\}, \quad \omega \in \Omega,
$$

where det stands for determinant. It is also an elementary fact that there exists a matrix $\tau_{*}(z) \in M_{n}(A)$ such that

$$
\left(z 1_{n}-\tau\right) \tau_{*}(z)=\tau_{*}(z)\left(z 1_{n}-\tau\right)=\delta\left(\operatorname{det}\left(z 1_{n}-\tau\right)\right)
$$

for each $z \in \mathbb{C}$.

Now, let $z \in \mathbb{C}$ be such that $\operatorname{det}\left(z 1_{n}-\tau(\omega)\right) \neq 0$ for all $\omega \in F$. We take a function $h \in A$ such that $h=1$ in a neighbourhood of $F$ and

$$
\operatorname{supp}(h) \subset\left\{\omega \in \Omega: \operatorname{det}\left(z 1_{n}-\tau(\omega)\right) \neq 0\right\} .
$$

Since $\operatorname{det}\left(z 1_{n}-\tau\right) \in A$ and $A$ has the property (ii) of Definition 1.1, the function $g(\omega)=h(\omega)\left(\operatorname{det}\left(z 1_{n}-\tau(\omega)\right)^{-1}\right.$ (equal to zero outside the set supp $\left.(h)\right)$ is an element of $A$. From (3.1) we deduce that

$$
\left(z 1_{n}-T\right) \Phi_{n}\left(g \tau_{*}(z)\right)=\Phi_{n}\left(g \tau_{*}(z)\right)\left(z 1_{n}-T\right)=\Phi_{n}(\delta(h)) .
$$

Since we have $\operatorname{supp}(1-h) \cap F=\varnothing$, it is clear that $\Phi_{n}(\delta(h)) \mid X_{\Phi}^{n}(F)$ is the identity on $X_{\Phi}^{n}(F)$. Therefore

$$
\Phi_{n}\left(g \tau_{*}(z)\right) \mid X_{\Phi}^{n}(F)=\left(\left(z 1_{n}-T\right) \mid X_{\Phi}^{n}(F)\right)^{-1},
$$

that is $z \notin \sigma\left(T \mid X_{\Phi}^{n}(F)\right)$.

Finally, if $\operatorname{det}\left(z 1_{n}-\tau(\omega)\right) \neq 0$ for all $\omega \in F$, then there exists a neighbourhood $V$ of $z$ such that if $w \in V$, then $\operatorname{det}\left(w 1_{n}-\tau(\omega)\right) \neq 0$ for all $\omega \in F$. Consequently, the set $\bigcup\{\sigma(\tau(\omega)): \omega \in F\}$ is closed.

3.2 Remark. The inclusion in the statement of Lemma 3.1 can be written as

$$
\sigma\left(T \mid X_{\Phi}^{n}(F)\right) \subset \bigcup\{\sigma(\tau(\omega)): \omega \in F \cap \operatorname{supp}(\Phi)\},
$$

using Corollary 2.3.

3.3 LEMMA. Let $L \subset \mathbb{C}$ be a closed set and let

$$
\theta(L)=\{\omega \in \Omega: \sigma(\tau(\omega)) \cap L \neq \varnothing\} .
$$

Then $\theta(L)$ is a compact subset of $\Omega$ with the property that $\sigma\left(T \mid X_{\Phi}^{n}(F)\right) \cap L=\varnothing$ whenever $\theta(L) \cap F=\varnothing, F$ closed in $\Omega$.

Proof. If $\omega_{0} \notin \theta(L)$, then $\sigma\left(\tau\left(\omega_{0}\right)\right) \cap L=\varnothing$. Thus, by the upper semicontinuity of the spectrum, there exists a neighbourhood $W_{0}$ of $\omega_{0}$ such that $\sigma(\tau(\omega)) \cap L=\varnothing$ for each $\omega \in W_{0}$. Hence $\Omega \backslash \theta(L)$ is open.

Now, let $F=\bar{F} \subset \Omega$ be such that $\theta(L) \cap F=\varnothing$. If $z$ were a point of $\sigma\left(T \mid X_{\Phi}^{n}(F)\right) \cap L$, then, by virtue of Lemma 3.1, there would exist a point $\omega \in F$ such that $z \in \sigma(\tau(\omega))$. Therefore $\omega \in \theta(L) \cap F$, which contradicts the choice of $F$.

3.4 Lemma. The operator T satisfies the condition $(\beta)$ of Bishop [4].

Proof. We have to show that if $U \subset \mathbb{C}$ is an arbitrary open set and $\left\{g_{p}\right\}_{p=1}^{\infty}$ is a sequence of $X^{n}$-valued functions, analytic in $U$, such that $\left(z 1_{n}-T\right) g_{p}(z) \rightarrow 0(p \rightarrow \infty)$ uniformly on the compact subsets of $U$, then it follows that $g_{p}(z) \rightarrow 0(p \rightarrow \infty)$ uniformly on the compact subsets of $U$. 
Let $\left\{g_{p}\right)_{p-1}^{\infty}$ be a sequence as above and let $\Delta \subset U$ be a fixed closed disc. We show that $g_{p}(z) \rightarrow 0(p \rightarrow \infty)$ uniformly on $\Delta$.

We consider the set $\theta(\Delta) \subset \Omega$ (defined in Lemma 3.3) and fix a point $\omega_{0} \in \theta(\Delta)$. Let $D_{0} \subset \bar{D}_{0} \subset U$ be an open disc containing $\Delta$ and let $V_{0} \subset \mathbb{C}$ be an open set such that $\bar{D}_{0} \cap \bar{V}_{0}=\varnothing$ and $\sigma\left(\tau\left(\omega_{0}\right)\right) \subset D_{0} \cup V_{0}$, which is obviously possible. By the upper semicontinuity of the spectrum, we infer the existence of an open neighbourhood $W_{0}$ of $\omega_{0}$ in $\Omega$ such that if $\omega \in W_{0}$, then $\sigma(\tau(\omega)) \subset D_{0} \cup V_{0}$. This procedure can be applied to any point $\omega$ of $\theta(\Delta)$. By the compactness of $\theta(\Delta)$ (Lemma 3.3), we obtain a finite open cover $\left\{W_{1}, \ldots, W_{m}\right\}$ of $\theta(\Delta)$, open discs $D_{1}, \ldots, D_{m}$ whose closures are in $U$ and open sets $V_{1}, \ldots, V_{m}$ in $\mathbb{C}$ such that $D_{q} \supset \Delta, \bar{D}_{q} \cap \bar{V}_{q}=\varnothing$ and $\sigma(\tau(\omega)) \subset D_{q} \cup V_{q}$ for every $\omega \in W_{q}(q=1, \ldots, m)$. Let $W_{m+1}=\Omega \backslash \theta(\Delta)$. We take the functions $h_{1}, \ldots, h_{m}, h_{m+1}$ from $A$ such that $h_{1}+\ldots+h_{m}+h_{m+1}=1$ and $\operatorname{supp}\left(h_{q}\right) \subset W_{q}$ $(q=1, \ldots, m+1)$. Then consider the matrices $\alpha_{q}=\delta\left(h_{q}\right)$. Note that

$$
\Phi_{n}\left(\alpha_{q}\right) g_{p}(z) \in X_{\Phi}^{n}\left(\operatorname{supp}\left(\alpha_{q}\right)\right), \quad q=1, \ldots, m, m+1,
$$

and that $\sigma\left(T \mid X_{\Phi}^{n}\left(\operatorname{supp}\left(\alpha_{q}\right)\right) \subset \bar{D}_{q} \cup \bar{V}_{q}(q=1, \ldots, m)\right.$. Since $\bar{D}_{q} \cap \bar{V}_{q}=\varnothing$, we can take another open disc $D_{q}^{\prime} \supset \bar{D}_{q}$ in $U$ such that $D_{q}^{\prime} \cap \bar{V}_{q}=\varnothing(1 \leqslant q \leqslant m)$. Note that the operator

$$
\left(z 1_{n}-T\right) \mid X_{\Phi}^{n}\left(\operatorname{supp}\left(\alpha_{q}\right)\right)
$$

is invertible for $z \in D_{q}^{\prime} \backslash \bar{D}_{q}$ and that $\Phi_{n}\left(\alpha_{q}\right)$ commutes with $T$. Therefore, as $p \rightarrow \infty$ $\Phi_{n}\left(\alpha_{q}\right) g_{p}(z) \rightarrow 0$ uniformly on the compact subsets of $D_{q}^{\prime}$. By the maximum principle, we deduce that $\Phi_{n}\left(\alpha_{q}\right) g_{p}(z) \rightarrow 0(p \rightarrow \infty)$ uniformly on $\bar{D}_{q}$, in particular on $\Delta$, for every $q=1, \ldots, m$.

From Lemma 3.3 we obtain that

$$
\sigma\left(T \mid X_{\Phi}^{n}\left(\operatorname{supp}\left(\alpha_{m+1}\right)\right) \cap \Delta=\varnothing .\right.
$$

Hence $\Phi_{n}\left(\alpha_{m+1}\right) g_{p}(z) \rightarrow 0(p \rightarrow \infty)$ uniformly on $\Delta$, and therefore

$$
g_{p}(z)=\Phi_{n}\left(\left(\alpha_{1}\right) g_{p}(z)+\ldots+\Phi_{n}\left(\alpha_{m+1}\right) g_{p}(z) \rightarrow 0 \quad(p \rightarrow \infty)\right.
$$

uniformly for $z \in \Delta$.

The general assertion now follows by covering an arbitrary compact subset $L \subset U$ with a finite number of closed discs and applying the previous argument to each of these discs.

Since $T$ satisfies the condition $(\beta)$, it follows that $T$ has the single-valued extension property. In particular, we can discuss the spectral spaces

$$
X_{T}^{n}(L)=\left\{x \in X^{n}: \gamma_{T}(x) \subset L\right\},
$$

where $L \subset \mathbb{C}$ is an arbitrary closed set and $\gamma_{T}(x)$ is the local spectrum of $T$ at $x$ (see [5] or [12] for details). In addition, the space $X_{T}^{n}(L)$ is closed (which is an easy consequence of the condition $(\beta)), X_{T}^{n}(L)$ is invariant under every operator that commutes with $T$ (that is $X_{T}^{n}(L)$ is hyperinvariant) and $\sigma\left(T \mid X_{T}^{n}(L)\right) \subset L[5,12]$.

\subsection{Lemma. The operator $T$ is decomposable.}

Proof. Let $\left\{U_{1}, U_{2}\right\}$ be an open cover of $\mathbb{C}$, and fix a point $\omega_{0} \in \Omega$. Then we can choose two open sets $V_{0}^{1}$ and $V_{0}^{2}$ in $\mathbb{C}$ such that $\sigma\left(\tau\left(\omega_{0}\right)\right) \subset V_{0}^{1} \cup V_{0}^{2}, \bar{V}_{0}^{g} \subset U_{a}$ $(q=1,2)$ and $\bar{V}_{0}^{1} \cap \bar{V}_{0}^{2}=\varnothing$. Let $W_{0} \subset \Omega$ be an open set such that $\omega \in \bar{W}_{0}$ implies that $\sigma(\tau(\omega)) \subset V_{0}^{1} \cup V_{0}^{2}$. Since $\Omega$ is compact, the previous remark shows that we can find 
an open cover $\left\{W_{1}, \ldots, W_{m}\right\}$ of $\Omega$ and open sets $\left\{V_{p}^{q}: 1 \leqslant p \leqslant m, q=1,2\right\}$ in $\mathbb{C}$ such that

(a) $\bar{V}_{p}^{q} \subset U_{q}, \bar{V}_{p}^{1} \cap \bar{V}_{p}^{2}=\varnothing$;

(b) if $\omega \in \bar{W}_{p}$ then $\sigma(\tau(\omega)) \subset \bar{V}_{p}^{1} \cup \bar{V}_{p}^{2}$

for all $p=1, \ldots, m$ and $q=1,2$. From Lemma 3.1 and the property (b) we deduce that

$$
\sigma\left(T \mid X_{\Phi}^{n}\left(\bar{W}_{p}\right)\right) \subset \bigcup_{\omega \in \bar{W}_{p}} \sigma(\tau(\omega)) \subset \bar{V}_{p}^{1} \cup \bar{V}_{p}^{2} .
$$

Therefore

$$
X_{\Phi}^{n}\left(\bar{W}_{p}\right) \subset X_{T}^{n}\left(\bar{V}_{p}^{1} \cup \bar{V}_{p}^{2}\right)
$$

by the fact that the space from the right-hand side is spectral maximal $[5,12]$. Let us also note the decomposition

$$
X_{T}^{n}\left(\bar{V}_{p}^{1} \cup \bar{V}_{p}^{2}\right)=X_{T}^{n}\left(\bar{V}_{p}^{1}\right)+X_{T}^{n}\left(\bar{V}_{p}^{2}\right),
$$

which follows from (a), the decomposition of the space with respect to separate parts of the spectrum (see, for instance, [12, Theorem III.3.11]) and the fact that all involved spaces are spectral maximal.

According to (2.2) and the above considerations, we can write

$$
X^{n}=\sum_{p=1}^{m} X_{\Phi}^{n}\left(\bar{W}_{p}\right)=\sum_{p=1}^{m} X_{T}^{n}\left(\bar{V}_{p}^{1}\right)+\sum_{p=1}^{m} X_{T}^{n}\left(\bar{V}_{p}^{2}\right)=X_{T}^{n}\left(\bar{U}_{1}\right)+X_{T}^{n}\left(\bar{U}_{2}\right),
$$

which proves the decomposability of $T$, by virtue of [12, Theorem IV.4.28].

3.6 Remark. Lemma 3.5 is also stated in [2] as Corollary 3.14, in a framework different from ours. It seems to us that the proof of the above-mentioned corollary needs our slightly stronger condition (ii) of Definition 1.1 rather than condition (b) from [2, Definition 3.1] (see the construction of the function $f$ [2, p. 304]). We are indebted to the referee for drawing our attention to this result from [2].

3.7 Corollary. If $\sigma(T)$ contains more than one point, then $T$ has at least one proper hyperinvariant subspace.

This fact is well known in the theory of decomposable operators and is based on the existence of a compact subset $L \subset \sigma(T)$ such that $X_{T}^{n}(L)$ is neither zero nor the whole space. As we have already mentioned, $X_{T}^{n}(L)$ is a hyperinvariant subspace of $T$.

3.8 Remark. If $A=C(\Omega)$ and $\Phi$ is obtained via a spectral measure on $\Omega$, then the operator $T$ is $n$-spectral [9]. If $\sigma(T)$ has more than one point, then $T$ has a proper hyperinvariant subspace, as proved in [9]. Consequently, Corollary 3.7 provides an extension of this result.

3.9 THEOREM. Every $(A, n)$-scalar operator is super-decomposable.

Proof. We use the notation and the discussion from the proof of Lemma 3.5.

Let $\left\{f_{1}, \ldots, f_{m}\right\} \subset A$ be such that $f_{1}+\ldots+f_{m}=1$ and $\operatorname{supp}\left(f_{p}\right) \subset W_{p}(p=1, \ldots, m)$. Let also $Q_{p}^{q}$ be the spectral projection of the space $X_{T}^{n}\left(\bar{V}_{p}^{1} \cup \bar{V}_{p}^{2}\right)$ onto $X_{T}^{n}\left(\bar{V}_{p}^{q}\right)$ 
$(q=1,2 ; p=1, \ldots, m)$, which is obtained from the decomposition (3.3) via the analytic functional calculus of the restriction of $T$ to $X_{T}^{n}\left(\bar{V}_{p}^{1} \cup \bar{V}_{p}^{2}\right)$ (see [12, Theorem III.3.11]). Since

$$
\Phi_{n}\left(\delta\left(f_{p}\right)\right) X^{n} \subset X_{\Phi}^{n}\left(\operatorname{supp}\left(f_{p}\right)\right) \subset X_{\Phi}^{n}\left(\bar{W}_{p}\right) \subset X_{T}^{n}\left(\bar{V}_{p}^{1} \cup \bar{V}_{p}^{2}\right),
$$

we may define the operators

$$
R_{q}=\sum_{p=1}^{m} Q_{p}^{q} \Phi_{n}\left(\delta\left(f_{p}\right)\right) \in \mathscr{L}\left(X^{n}\right), \quad q=1,2 .
$$

It is easily seen that $R_{1}+R_{2}=1_{n}$. Moreover,

$$
\begin{aligned}
T R_{q} & =T\left(\sum_{p=1}^{m} Q_{p}^{q} \Phi_{n}\left(\delta\left(f_{p}\right)\right)\right. \\
& =\sum_{p=1}^{m}\left(T \mid X_{T}^{n}\left(\bar{V}_{p}^{1} \cup \bar{V}_{p}^{2}\right)\right) Q_{p}^{q} \Phi_{n}\left(\delta\left(f_{p}\right)\right) \\
& =\sum_{p=1}^{m} Q_{p}^{q}\left(T \mid X_{T}^{n}\left(\bar{V}_{p}^{1} \cup \bar{V}_{p}^{2}\right)\right) \Phi_{n}\left(\delta\left(f_{p}\right)\right) \\
& =\sum_{p=1}^{m} Q_{p}^{q} T \Phi_{n}\left(\delta\left(f_{p}\right)\right)=R_{q} T,
\end{aligned}
$$

since every operator commutes with its analytic functional calculus and $\delta\left(f_{p}\right)$ is in the centre of $M_{n}(A)$. We also have

$$
R_{q}\left(X^{n}\right) \subset \sum_{p=1}^{m} X_{T}^{n}\left(\bar{V}_{p}^{q}\right) \subset X_{T}^{n}\left(\bar{U}_{q}\right),
$$

which insures, by virtue of [8, Theorem 1.4], that $T$ is super-decomposable. The proof of the theorem is complete.

The authors thank the referee for suggesting the use of [8, Theorem 1.4] to shorten the original proof of Theorem 3.9.

3.10 Remark. Let $\Omega_{\tau}=\tau(\Omega)$ and set $A_{\tau}=\left\{f \in C\left(\Omega_{\tau}\right): f \circ \tau \in A\right\}$, which is a subalgebra of $C\left(\Omega_{\tau}\right)$. Then the map $\Phi_{\tau}: A_{\tau} \rightarrow \mathscr{L}(X)$ given by $\Phi_{\tau}(f)=\Phi(f \circ \tau)$ is a unital algebra morphism. Suppose that $A_{\mathrm{t}}$ has the properties (i) and (ii) from Definition 1.1 (this happens, for instance, when $A=C(\Omega)$ ). Then the morphism $\Phi_{\tau}$ can be used instead of $\Phi$. In this case there is no loss of generality in assuming that $\Omega$ is a compact subset of $\mathbb{C}^{n^{2}}$ and that $\tau$ is the matrix of the coordinate functions on $\mathbb{C}^{n^{2}}$, restricted to $\Omega$.

\section{More about the spectrum}

In this section we assume that $A \subset C(\Omega)$ has the properties (i), (ii) and (iii) of Definition 1.1. As in the previous section, we fix a unital algebra morphism $\Phi: A \rightarrow \mathscr{L}(X)$, an element $\tau=\left(\tau_{j k}\right)_{j, k=1}^{n} \in M_{n}(A)$, and consider the $(A, n)$-scalar operator $T=\Phi_{n}(\tau) \in \mathscr{L}\left(X^{n}\right)$.

For every closed set $F \subset \Omega$ we define the set

$$
S_{\tau, F}=\bigcup_{\omega \in F} \sigma(\tau(\omega)) \subset \mathbb{C} .
$$


The set $S_{\tau, F}$ is closed (in fact compact), by Lemma 3.1. When $F=\operatorname{supp}(\Phi)$, the set $S_{\tau, F}$ will be denoted simply by $S_{\tau}$.

4.1 LeMma. For every $h \in A$, there is an analytic function $\phi_{h}: \mathbb{C} \backslash S_{\tau, F} \rightarrow M_{n}(A)$ such that $\left(z 1_{n}-\tau\right) \phi_{h}(z)=\delta(h)$ for all $z \notin S_{\tau, F}$, where $F=\operatorname{supp}(h)$.

Proof. Consider the Banach space $Y=A^{n}$ and the map $\Psi: A \rightarrow \mathscr{L}(Y)$ given by

$$
\Psi(h) f_{1} \oplus \ldots \oplus f_{n}=h f_{1} \oplus \ldots \oplus h f_{n}, \quad h, f_{1}, \ldots, f_{n} \in A .
$$

Clearly, $\Psi$ is a unital algebra morphism. Let $\Psi_{n}: M_{n}(A) \rightarrow \mathscr{L}\left(Y^{n}\right)$ be the unital algebra morphism induced by $\Psi$. If we identify $Y^{n}$ with $M_{n}(A)$, then, with this identification, $\Psi_{n}(\alpha) \beta=\alpha \beta$ for all $\alpha, \beta \in M_{n}(A)$. In particular $\Psi_{n}(\tau)$ is the multiplication by the matrix $\tau$, which will also be denoted by $\tau$. The operator $\tau$ is $(A, n)$-scalar, and therefore it has the properties described in the previous section.

It is easily seen that $\delta(h) \in Y_{\Psi}^{n}(\operatorname{supp}(h))$ (which is defined by (2.1)). According to Lemma 3.1,

$$
\sigma\left(\tau \mid Y_{\Psi}^{n}(F)\right) \subset S_{\tau, F},
$$

where $F=\operatorname{supp}(h)$. Consequently, we may take

$$
\phi_{h}(z)=\left(\left(z 1_{n}-\tau\right) \mid Y_{\Psi}^{n}(F)\right)^{-1} \delta(h), \quad z \notin S_{\tau, F} .
$$

4.2 Lemma. Assume that there exists a compact subset $L \subset S_{\tau} \backslash \sigma(T)$ such that $S_{\tau} \backslash L$ is also compact. Then $L=\varnothing$.

Proof. Let us assume that $L \neq \varnothing$. Let $V_{1} \supset L$ and $V_{2} \supset S_{\tau} \backslash L$ be open sets such that $\bar{V}_{1} \cap \bar{V}_{2}=\varnothing$. Then there is an open neighbourhood $W$ of $\operatorname{supp}(\Phi)$ such that $S_{\tau, \bar{W}} \subset V_{1} \cup V_{2}$. We may also assume that $\Gamma=\partial V_{1}$ is a finite system of Jordan rectifiable curves, positively oriented.

Let $h \in A$ be such that $h=1$ in a neighbourhood of $\operatorname{supp}(\Phi)$ and $\operatorname{supp}(h) \subset W$. Also let $\phi_{h}$ be the analytic function given by Lemma 4.1, which is defined outside the set $S_{\tau, \bar{w}}$. Then we may consider the element

$$
e=\frac{1}{2 \pi i} \int_{\Gamma} \phi_{h}(z) d z \in M_{n}(A) .
$$

Set $F_{1}=\{\omega \in \Omega: h(\omega)=1\}$. Since $\delta(h)(\omega)=1_{n}$ for $\omega \in F_{1}, \phi_{h}(z)(\omega)=\left(z 1_{n}-\tau(\omega)\right)^{-1}$. It follows from our assumption on the algebra $A$ (Definition 1.1(iii)) that the point evaluations are continuous. Hence

$$
e(\omega)=\frac{1}{2 \pi i} \int_{\Gamma}\left(z 1_{n}-\tau(\omega)\right)^{-1} d z, \quad \omega \in F_{1},
$$

which shows that $e(\omega)^{2}=e(\omega)$ (in fact $e(\omega)$ is a spectral projection of $\tau(\omega)$ ). Since $F_{1}$ is a neighbourhood of $\operatorname{supp}(\Phi)$, it follows that $\Phi_{n}(e)$ is an idempotent. In addition, $\Phi_{n}(e)$ commutes with $T$ because of the equality $\tau(\omega) e(\omega)=e(\omega) \tau(\omega)\left(\omega \in F_{1}\right)$.

Consider now the integral

$$
e_{w}=\frac{1}{2 \pi i} \int_{\Gamma}(w-z)^{-1} \phi_{h}(z) d z, \quad w \notin \bar{V}_{1} .
$$


It is clear that

$$
\left(w 1_{n}-\tau(\omega)\right) e_{w}(\omega)=e_{w}(\omega)\left(w 1_{n}-\tau(\omega)\right)=e(\omega)
$$

for all $\omega \in F_{1}$ and $w \notin \vec{V}_{1}$.

Since $\Phi_{n}(e)$ is idempotent, it follows that $Z=\Phi_{n}(e)\left(X^{n}\right)$ is a closed subspace of $X^{n}$, invariant under $T$ and also under $\Phi_{n}\left(e_{w}\right)$. Moreover, from (4.2) we deduce that

$$
\left(\left(w 1_{n}-T\right) \mid Z\right)\left(\Phi_{n}\left(e_{w}\right) \mid Z\right)=\left(\Phi_{n}\left(e_{w}\right) \mid Z\right)\left(\left(w 1_{n}-T\right) \mid Z\right)=1_{z},
$$

where $1_{Z}$ is the identity of $Z$. This shows that $\sigma(T \mid Z) \subset \bar{V}_{1}$. On the other hand, $\sigma(T) \subset V_{2}$, by Remark 3.2 and the property of $L$. Therefore $\sigma(T) \cap \sigma(T \mid Z)=\varnothing$, which is not possible unless $Z=\{0\}$. This shows that $\Phi_{n}(e)=0$, so that $e(\omega)=0$ for each $\omega \in \operatorname{supp}(\Phi)$, by virtue of Lemma 2.5 , which contradicts our assumption. Indeed, if $z_{0} \in L$, then there exists $\omega_{0} \in \operatorname{supp}(\Phi)$ such that $z_{0} \in \sigma\left(\tau\left(\omega_{0}\right)\right)$. Then $V_{1}$ contains at least one point from the spectrum of the matrix $\tau\left(\omega_{0}\right)$, whence $e\left(\omega_{0}\right) \neq 0$. Consequently we must have $L=\varnothing$.

\subsection{Lemma. Let $F \subset \Omega$ be closed and let}

$$
X_{\Phi}(F)=\bigcap\{\operatorname{ker}(\Phi(f)): \operatorname{supp}(f) \cap F=\varnothing\} .
$$

Then the space $X_{\Phi}(F)^{n}$ is invariant under $T$ and the restriction $T \mid X_{\Phi}(F)^{n}$ is $(A, n)$ scalar.

Proof. It is easily seen that $X_{\Phi}(F)^{n}=X_{\Phi}(F) \oplus \ldots \oplus X_{\Phi}(F)$ ( $n$ copies) is invariant under $T$. Since $X_{\Phi}(F)$ is invariant under $\Phi(f)$ for every $f \in A$, we may define the map

$$
A \ni f \longrightarrow \Phi_{F}(f)=\Phi(f) \mid X_{\Phi}(F) \in \mathscr{L}\left(X_{\Phi}(F)\right),
$$

which is a unital algebra morphism. If $\Phi_{F, n}$ is the unital algebra morphism from $M_{n}(A)$ into $\mathscr{L}\left(X_{\Phi}(F)^{n}\right)$ induced by $\Phi_{F}$, then $T \mid X_{\Phi}(F)^{n}=\Phi_{F, n}(\tau)$, which is precisely our assertion.

4.4 REMARK. With the notation of Lemma 4.3, we have the inclusion $\sigma\left(T \mid X_{\Phi}(F)^{n}\right) \subset \sigma(T)$. Indeed, if $z \notin \sigma(T)$, then the space $X_{\Phi}(F)^{n}$ is invariant under $\left(z 1_{n}-T\right)^{-1}$, since

$$
\Phi_{n}(\delta(f))\left(z 1_{n}-T\right)^{-1} x=\left(z 1_{n}-T\right)^{-1} \Phi_{n}(\delta(f)) x=0
$$

for every $f \in A$ with $\operatorname{supp}(f) \cap F=\varnothing$, and each $x \in X_{\Phi}(F)^{n}$.

4.5 Lemma. The morphism $\Phi_{F}$ from (4.3) has the following property:

$$
\operatorname{int}(F) \cap \operatorname{supp}(\Phi) \subset \operatorname{supp}\left(\Phi_{F}\right) \subset F \cap \operatorname{supp}(\Phi)
$$

for each closed $F$.

Proof. Let $X_{F}$ be the space $X_{\Phi}(F)$, defined in Lemma 4.3. Let also $f \in A$ be such that $\operatorname{supp}(f) \cap F \cap \operatorname{supp}(\Phi)=\varnothing$. By using the normality of the algebra $A$, we can write $f=f_{1}+f_{2}$, where $f_{1}, f_{2} \in A$, $\operatorname{supp}\left(f_{1}\right) \cap F=\varnothing$ and $\operatorname{supp}\left(f_{2}\right) \cap \operatorname{supp}(\Phi)=\varnothing$. Then

$$
\Phi_{F}(f)=\Phi\left(f_{1}\right)\left|X_{F}+\Phi\left(f_{2}\right)\right| X_{F}=0,
$$

which shows that $\operatorname{supp}\left(\Phi_{F}\right) \subset F \cap \operatorname{supp}(\Phi)$. 
Conversely, let $\omega_{0} \in \operatorname{int}(F) \cap \operatorname{supp}(\Phi)$, let $W_{0}$ be an open neighbourhood of $\omega_{0}$ such that $\bar{W}_{0} \subset \operatorname{int}(F)$, let $W_{1}=\operatorname{int}(F)$ and let $W_{2} \subset \Omega$ be open such that $\bar{W}_{2} \cap \bar{W}_{0}=\varnothing$ and $W_{1} \cup W_{2}=\Omega$. Then, by Proposition 2.1 (with $n=1$ ),

$$
X=X_{\bar{W}_{1}}+X_{\bar{W}_{2}}=X_{F}+X_{\bar{w}_{2}} .
$$

If $f \in A$ and $\operatorname{supp}(f) \subset W_{0}$, then $\Phi(f) \mid X_{\bar{W}_{2}}=0$. Since $\omega_{0} \in \operatorname{supp}(\Phi)$, this shows that $\omega_{0} \in \operatorname{supp}\left(\Phi_{F}\right)$.

4.6 THEOREM. Let $T \in \mathscr{L}\left(X^{n}\right)$ be an $(A, n)$-scalar operator such that $T=\Phi_{n}(\tau)$. Then we have the equality

$$
\sigma(T)=\bigcup\{\sigma(\tau(\omega)): \omega \in \operatorname{supp}(\Phi)\}
$$

Proof. The inclusion $\sigma(T) \subset S_{\tau}$ has already been noted (see Remark 3.2).

Conversely, assume that there exists a point $z_{0} \in S_{\tau} \backslash \sigma(T)$. Let $\omega_{0} \in \Omega$ be such that $z_{0} \in \sigma(\tau(\omega))$. Let $V_{1}, V_{2}$ be open sets in $\mathbb{C}$ such that $V_{1} \ni z_{0}, V_{2} \supset \sigma(T), \bar{V}_{1} \cap \bar{V}_{2}=\varnothing$ and $\sigma\left(\tau\left(\omega_{0}\right)\right) \subset V_{1} \cup V_{2}$. Then there exists an open set $W_{0} \ni \omega_{0}$ in $\Omega$ such that $\sigma(\tau(\omega)) \subset V_{1} \cup V_{2}$ for every $\omega \in F=\bar{W}_{0}$. According to Remark 4.4, we have the inclusion $\sigma\left(T_{F}\right) \subset \sigma(T) \subset V_{2}$, where $T_{F}=T \mid X_{\oplus}(F)^{n}$. On the other hand,

$$
\bigcup\left\{\sigma(\tau(\omega)): \omega \in \operatorname{supp}\left(\Phi_{F}\right)\right\} \subset S_{\tau, \Phi} \subset V_{1} \cup V_{2},
$$

by virtue of Lemma 4.5. From the same lemma it also follows that $\omega_{0} \in \operatorname{supp}\left(\Phi_{F}\right)$. This shows that the set

$$
L=\bigcup\left\{\sigma(\tau(\omega)): \omega \in \operatorname{supp}\left(\Phi_{F}\right) \cap \bar{V}_{1}\right\}
$$

is non-empty, which contradicts Lemma 4.2, applied to $T_{F}$. Therefore $S_{\tau} \backslash \sigma(T)=\varnothing$.

4.7 Definition. The map $\Phi_{n}: M_{n}(A) \rightarrow \mathscr{L}\left(X^{n}\right)$ is said to be of finite algebraic order if there exists an integer $m \geqslant 1$ such that from the fact that $\alpha(\omega)=0$ for all $\omega \in \operatorname{supp}\left(\Phi_{n}\right)$ and a certain $\alpha \in M_{n}(A)$, it follows that $\Phi_{n}\left(\alpha^{m}\right)=0$.

If $A=C^{\gamma}(\Omega)$ and $\Phi_{n}: M_{n}(A) \rightarrow \mathscr{L}\left(X^{n}\right)$ is continuous, then for every $\beta \in M_{n}(A)$ which is null on $\operatorname{supp}\left(\Phi_{n}\right)$ together with its partial derivatives up to order $r$, we have $\Phi_{n}(\beta)=0$. This fact is well known for scalar distributions and can be extended to vector distributions as well; an outline of the proof can be found in [12, Lemma IV.8.8]. This shows, in particular, that $\Phi_{n}$ is of finite algebraic order $\leqslant r+1$.

We can now complete Corollary 3.7 with the following statement.

4.8. CoRollary. If $\sigma(T)=\left\{z_{0}\right\}$ and the morphism $\Phi_{n}: M_{n}(A) \rightarrow \mathscr{L}\left(X^{n}\right)$ is of finite algebraic order, then $z_{0} 1_{n}-T$ is nilpotent.

In particular, if $T$ is not a multiple of the identity, then $T$ has a proper hyperinvariant subspace.

Proof. It follows from Theorem 4.6 that $\sigma(\tau(\omega))=\left\{z_{0}\right\}$ for every $\omega \in \operatorname{supp}(\Phi)$. In other words, the matrix $z_{0} 1_{n}-\tau(\omega)$ is nilpotent for each $\omega \in \operatorname{supp}(\Phi)$, that is $\left(z_{0} 1_{n}-\tau(\omega)\right)^{n}=0(\omega \in \operatorname{supp}(\Phi))$.

Since the map $\Phi_{n}$ is of finite algebraic order, $\Phi_{n}\left(\left(z_{0} 1_{n}-\tau\right)^{m n}\right)=0$ for some integer $m \geqslant 1$, that is $z_{0} 1_{n}-T$ is nilpotent. If $T$ is not a multiple of the identity, then $\operatorname{ker}\left(z_{0} 1_{n}-T\right)$ is a proper hyperinvariant subspace of $T$. 
Note added in proof. E. Albrecht (in a private communication) has shown that [2, Definition 3.1, conditions (a) and (b)] imply Definition 1.1 (i) and (ii) above. The converse is also true (see Remark 3.6).

\section{References}

1. E. Albrecht and S. Frunză, 'Non-analytic functional calculi in several variables', Manuscripta Math. 32 (1980) 263-294.

2. E. AlbreCht and R. D. Mehta, 'Some remarks on local spectral theory', J. Operator Theory 12 (1984) $285-317$.

3. C. Apostol, 'Spectral decompositions and functional calculus', Rev. Roumaine Math. Pures Appl. 13 (1968) $1481-1528$.

4. E. Bishop, 'A duality theorem for an arbitrary operator', Pacific J. Math. 9 (1959) 379-394.

5. I. COLOJOARĂ and C. FoIAŞ, Theory of generalized spectral operators (Gordon and Breach, New York, 1968).

6. R. G. Douglas and C. PearCY, 'Hyperinvariant subspaces and transitive algebras', Michigan Math J. 19 (1972) $1-12$.

7. T. B. Hoover, 'Hyperinvariant subspaces for $n$-normal operators', Acta Sci. Math. (Szeged) 32 (1971) 109-119.

8. K. B. Laursen and M. M. NeumanN, 'Decomposable operators and automatic continuity', $J$. Operator Theory 15 (1986) 33-51.

9. M. OMLadič, 'On N-spectral operators', Rev. Roumaine Math. Pures Appl. to appear.

10. M. ORHON, 'Algebras of operators containing a Boolean algebra of projections of finite multiplicity', Proceedings of the tenth operator theory conference, Bucharest, 1985.

11. H. RADJAVI and P. RoSENTHAL, 'Hyperinvariant subspaces for spectral and $n$-normal operators', Acta Sci. Math. (Szeged) 32 (1971) 121-126.

12. F.-H. VASILESCU, Analytic functional calculus and spectral decompositions (Editura Academiei and D. Reidel Publishing Company, Bucharest and Dordrecht, 1982).

\section{Department of Mathematics}

The National Institute for Scientific and Technical Creation

Bdul Păcii 220

79622 Bucharest

Romania 